\title{
Expression of B-cell translocation gene 2 is associated with favorable prognosis in hepatocellular carcinoma patients and sensitizes irradiation-induced hepatocellular carcinoma cell apoptosis in vitro and in nude mice
}

\author{
YUANYUAN CHEN, CHUAN CHEN, ZHIMIN ZHANG, HE XIAO, BIJING MAO, HUAN HUANG, \\ CHENCHEN DING, LIN LEI, HUI ZHANG, JIAN LI, MEI JIANG and GE WANG \\ Cancer Center, Institute of Surgical Research, Daping Hospital, The Third Military Medical University, \\ Chongqing 400042, P.R. China
}

Received July 1, 2015; Accepted July 12, 2016

DOI: $10.3892 / 01.2017 .5685$

\begin{abstract}
B-cell translocation gene 2 (BTG2) proteins have been reported to be putative tumor suppressors in various cancer types. The present study first assessed BTG2 expression in 44 human liver cancer tissue specimens, then investigated BTG2 expression in the regulation of hepatocellular carcinoma (HCC) cell apoptosis with or without radiotherapy in vitro and in vivo. The results revealed that BTG2 protein expression was significantly reduced in HCC tissues, and associated with better survival for HCC patients $(\mathrm{P}=0.05)$. BTG2 overexpression also sensitized Huh7 cells to radiation-induced apoptosis in vitro and in a nude mouse model, although restoration of BTG2 expression per se did not affect the viability and apoptosis of HCC cells. Future studies would confirm the role of BTG2 in hepatoma, and further develop BTG2 as a therapeutic strategy for controlling HCC.
\end{abstract}

\section{Introduction}

Hepatocellular carcinoma (HCC) is the third leading cause of cancer-related mortality, and ranks sixth among the most common cancers in the world (1-3). HCC is usually diagnosed at the advanced stage due to the lack of early detection methods

Correspondence to: Dr Ge Wang, Cancer Center, Institute of Surgical Research, Daping Hospital, The Third Military Medical University, 10 Changjiang Branch Road, Chongqing 400042, P.R. China

E-mail: wangge70@hotmail.com

Abbreviations: HCC, hepatocellular carcinoma; BTG2, B-cell translocation gene 2; Bax, Bcl2-associated X; TUNEL, terminal deoxynucleotidyl transferase-mediated dUTP nick-end labeling

Key words: hepatocellular carcinoma, BTG2, prognosis, apoptosis, radiotherapy, tumor suppressor gene such as serum biomarkers, or clinical symptoms and signs; this means that surgical cure is impossible. Overall, the prognosis of patients with HCC is extremely poor. Clinically, HCC patients are treated with surgical resection of tumor lesions, liver transplantation, chemotherapy or radiotherapy. Radiation therapy is being integrated with other treatment options in comprehensive treatment regimes for $\mathrm{HCC}$, in particular for localized hepatic tumors refractory to conventional therapy (4). To date, controlling HCC using radiation therapy remains unsatisfactory due to the low radiation sensitivity and tolerance of the whole liver (5). Thus, improving the therapeutic effect of radiation on hepatoma has clinical significance and is an urgent requirement.

Previously, tumor suppressor gene p53 has been used to enhance radiotherapy effectiveness in oral cancer patients following resection surgery of tumor lesions $(6,7)$. B-cell translocation gene 2 (BTG2) proteins were reported to be putative tumor suppressor genes, and constitute a p53-dependent component of the DNA damage cellular response pathway (8-10). BTG2 is an early growth response gene belonging to the anti-proliferative family (11-13). Previous studies indicate that the BTG2 protein functions to regulate cell differentiation, development and apoptosis (14,15). BTG2 expression has been demonstrated to be reduced or lost in a variety of cancers $(8,16-20)$. The restoration of BTG2 expression in a medulloblastoma mouse model suppressed tumor formation and growth (21). Since p53 plays a significant role in HCC development and progression, we first assessed BTG2 expression and its role in liver cancer. This is expected to provide insightful information on the association of BTG2 with HCC prognosis and promotion in HCC cell apoptosis following radiotherapy in vitro and in a nude mouse model of transplanted hepatoma.

\section{Materials and methods}

Patients and liver sample collection. All HCC tissue samples were collected from 44 patients in Daping Hospital (Chongqing, China). These patients were histologically diagnosed with 
HCC according to the World Health Organization (WHO) criteria (22). None of the patients underwent chemotherapy or radiotherapy prior to surgery. The study was approved by the ethics committee of Daping Hospital, The Third Military Medical University (Chongqing, China). Formalin-fixed and paraffin-embedded tissue blocks were retrieved from the Department of Pathology, and subjected to immunohistochemical analysis of BTG2 expression.

Immunohistochemistry. Immunohistochemical analysis of BTG2 expression was conducted according to a previous study using a standard avidin-biotin complex (ABC) method (22). Tissue sections were incubated with various antibodies at a dilution of 1:1,000 (GenWay Biotech Inc., San Diego, CA, USA) and at a dilution of 1:50 for Ki67 (BD Biosciences, Franklin Lakes, NJ, USA). Then, stained sections were independently evaluated and scored under a microscope by two pathologists using the following four-point scale (positive cell count, grades $0-3$ ): 0 , no positive cells; $1,<25 \%$ positive cells; $2,25-50 \%$ positive cells; $3,>50 \%$ positive cells. Low expression levels in the nuclei were defined as having an immunohistochemical staining score of $0-1$, whereas high expression levels in the nuclei were defined as having an immunohistochemical staining score of 2-3.

Cell line, culture and pEGFP-N1-BTG2 transfection. The human HCC cell line Huh7 was obtained from the American Type Culture Collection (Manassas, VA, USA), and maintained in Dulbecco's modified Eagle's medium (DMEM) supplemented with $10 \%$ fetal bovine serum (Invitrogen Life Technologies, Carlsbad, CA, USA), penicillin (100 U/ml; Sigma-Aldrich, St. Louis, MO, USA) and streptomycin $(100 \mu \mathrm{g} / \mathrm{ml}$, Sigma-Aldrich) in a humidified incubator with $5 \% \mathrm{CO}_{2}$ at $37^{\circ} \mathrm{C}$. For gene transfection, Huh7 cells in the logarithmic growth phase were digested with trypsin, counted, seeded at an appropriate density in six-well plates, and cultured in an incubator at $37^{\circ} \mathrm{C}$ with $5 \% \mathrm{CO}_{2}$. The next day, cells at $70-90 \%$ confluence were changed to serum-free DMEM culture, and transfected with eukaryotic expression vector pEGFP-N1-BTG2 or empty vector as a control using Lipofectamine 2000 (Invitrogen Life Technologies). After $24 \mathrm{~h}$, transfected cells were plated in a 12 -well plate at a 1:20 dilution ratio. The medium was replaced with complete medium containing G418 $(800 \mu \mathrm{g} / \mathrm{ml})$. Stable positive clones were selected 21 days later, and BTG2 protein expression was assessed using western blot analysis. Then, single clones were picked, evaluated and frozen to be used for further experiments.

Protein extraction and western blot analysis. Total cellular protein was extracted from cultured cells using lysis buffer, and subjected to western blot analysis as previously described (23). Dilutions of primary antibodies were as follows: BTG2 at a dilution of 1:1,000 (Sigma-Aldrich), Bax at a dilution of 1:1,000 (Abcam, Cambridge, MA, USA) and $\beta$-actin at a dilution of 1:10,000 (Abcam). Western blots were quantified using ImageJ software (National Institutes of Health, Bethesda, MD, USA).

Flow cytometric analysis of apoptosis. Apoptotic cells were detected using an Annexin V-FITC apoptosis detection kit
(BD Pharmingen, San Diego, CA, USA). Specifically, Huh7 cells transfected with empty vector or BTG2 expression vector were first seeded in six-well culture plates and cultured for $48 \mathrm{~h}$, then irradiated on the third day at a dose of $8 \mathrm{~Gy}$. After $24 \mathrm{~h}$, cells were harvested and washed twice with ice-cold phosphate-buffered saline, then centrifuged and resuspended with $1 \mathrm{X}$ binding buffer. A $100-\mu 1$ cell solution was transferred into a 5 -ml tube, then $5 \mu \mathrm{l}$ Annexin V-FITC and $5 \mu \mathrm{l}$ propidium iodide were added, respectively. After incubating for 15 min in the dark, samples were analyzed by flow cytometry (BD FACSCalibur, BD Biosciences) within one hour. Experiments were independently repeated at least three times.

In vitro cell counting assay. Huh7 cells with empty vector or pEGFP-N1-BTG2 transfection were seeded into 12-well culture plates at a density of $5 \times 10^{4}$ cells $/ \mathrm{ml}$ in triplicate, and grown overnight. The following day, cells were irradiated at a dose of $8 \mathrm{~Gy}$. The number of cells was then counted daily. Cell growth curves were plotted using the average of three independent experiments.

Animal experiments. Severe combined immune deficient mice were purchased from the Chinese Academy of Medical Sciences (Beijing, China), and housed and maintained in laminar flow cabinets under specific pathogen-free conditions. Animal use and experimental protocols were reviewed and approved by the ethics committee of the Third Military Medical University, Chongqing, China. For animal experiments, mice were first implanted with $1 \times 10^{7}$ Huh7 cells stably transfected with empty vector or $\mathrm{pEGFP-N1-BTG2} \mathrm{vector.} \mathrm{Following} \mathrm{tumor} \mathrm{cell} \mathrm{injec-}$ tion, the site was irradiated with 8 Gy on days 21,24 and 27. Tumor volume was measured twice a week for four weeks, and at the end of the experiments, xenograft tissues were removed and subjected to hematoxylin and eosin (H\&E), Bax, Ki67 and terminal deoxynucleotidyl transferase-mediated dUTP nick-end labeling (TUNEL) staining.

TUNEL staining. Apoptosis in xenograft tissues was analyzed by the TUNEL method. Formalin-fixed and paraffin-embedded xenograft sections were deparaffinized in xylene, rehydrated in a series of ethanol solutions, and then treated with $0.2 \mathrm{~N}$ $\mathrm{HCl}$ for $10 \mathrm{~min}$ at room temperature and with proteinase $\mathrm{K}$ $(20 \mu \mathrm{g} / \mathrm{ml})$ for $30 \mathrm{~min}$ at $37^{\circ} \mathrm{C}$. The sections were incubated in a $3 \%$ methanolic hydrogen peroxide solution for $5 \mathrm{~min}$ at room temperature to block potential endogenous peroxidase activity, and were further incubated with terminal deoxynucleotidyl transferase (TdT) buffer (Trevigen Inc., Gaithersburg, MA, USA) for $5 \mathrm{~min}$. The sections were transferred into a TdT enzyme mixture containing TdT buffer, TdT diluted at 1:400, biotinylated dUTP diluted at 1:200 (Roche, Indianapolis, IN, USA), and $400 \mu \mathrm{M}$ of $\mathrm{MnSO}_{4}$, then incubated at $37^{\circ} \mathrm{C}$ for 60 min. Then, an ABC kit (Vector Laboratories, Burlingame, CA, USA) was used to detect positive signals by incubating the sections with ABC solution for $30 \mathrm{~min}$ in the dark, and the sections were incubated with a 3-amino-9-ethylcarbazole (Sigma-Aldrich) solution for $30 \mathrm{~min}$ to visualize the peroxidase complex. Negative control sections were not incubated with deoxynucleotidyl transferase. Stained sections were independently reviewed by two pathologists with an Olympus microscope (Olympus Corporation, Tokyo, Japan). The 
Table I. Association of survival with BTG2 expression and clinicopathological data from HCC patients.

\begin{tabular}{|c|c|c|c|c|}
\hline \multirow[b]{2}{*}{ Clinicopathological features } & \multicolumn{2}{|c|}{ BTG2 expression } & \multirow[b]{2}{*}{$\chi^{2}$} & \multirow[b]{2}{*}{ P-value } \\
\hline & High & Low & & \\
\hline Age (years) & & & 1.462 & 0.227 \\
\hline$<60$ & 11 & 23 & & \\
\hline$\geq 60$ & 6 & 4 & & \\
\hline Gender & & & & $0.359^{\mathrm{a}}$ \\
\hline Male & 14 & 25 & & \\
\hline Female & 3 & 2 & & \\
\hline Pathology grade & & & 8.974 & $0.010^{\mathrm{a}}$ \\
\hline I & 1 & 5 & & \\
\hline II & 8 & 20 & & \\
\hline III & 8 & 2 & & \\
\hline pT & & & 1.365 & $0.674^{\mathrm{a}}$ \\
\hline $\mathrm{T} 1$ & 14 & 23 & & \\
\hline $\mathrm{T} 2$ & 2 & 1 & & \\
\hline $\mathrm{T} 3$ & 1 & 3 & & \\
\hline Regional lymph node status & & & & $1.000^{\mathrm{a}}$ \\
\hline No & 17 & 26 & & \\
\hline N1 & 0 & 1 & & \\
\hline $\mathrm{N} 2$ & 0 & 0 & & \\
\hline Metastasis status & & & & $1.000^{\mathrm{a}}$ \\
\hline M0 & 17 & 26 & & \\
\hline M1 & 0 & 1 & & \\
\hline Stage & & & 2.265 & $0.626^{\mathrm{a}}$ \\
\hline I & 14 & 21 & & \\
\hline II & 2 & 1 & & \\
\hline III & 1 & 3 & & \\
\hline IV & 0 & 2 & & \\
\hline \multirow[t]{3}{*}{$\operatorname{mOS}(95 \% \mathrm{CI})$} & & & 3.856 & $0.050^{\mathrm{b}}$ \\
\hline & 16.0 & 43.0 & & \\
\hline & $(1.210-30.790)$ & $(25.794-60.025)$ & & \\
\hline
\end{tabular}

${ }^{a}$ Fisher's exact probability test; ${ }^{b}$ log-rank test; BTG2, B-cell translocation gene 2; HCC, hepatocellular carcinoma; mOS, median overall survival; CI, confidence interval.

percentage of positive cells was calculated after counting the total cells and positively stained cells.

Statistical analysis. The data are expressed as the means \pm standard deviation, and analyzed using the independent-samples Student's t-test (two-tailed) with SPSS software (SPSS Inc., Chicago, IL, USA). Survival curves were calculated using the Kaplan-Meier method, and analyzed using the Cox regression test. $\mathrm{P} \leq 0.05$ was considered to indicate a statistically significant difference.

\section{Results}

BTG2 expression in HCC tissues is associated with increased survival rate in HCC patients. In this study, $44 \mathrm{HCC}$ tissue specimens were collected for immunohistochemical analysis of
BTG2 expression. Patients were divided into two groups (a low expression and high expression group) based on the staining data. Our results revealed that patients with higher BTG2 expression in the nucleus had better survival rates, compared with patients with low BTG2 expression (Fig. 1 and Table I).

BTG2 overexpression sensitizes Huh7 cells to radiation-induced apoptosis in vitro. Next, the in vitro effects of BTG2 expression on HCC cells were assessed by stably overexpressing BTG2 in Huh7 cells. Following stable gene transfection, western blot analysis data demonstrated that BTG2 expression was increased 3.6-fold $(\mathrm{P}<0.05)$ at protein level in stably transfected Huh7-BTG2 cells, compared with Huh7 cells (Fig. 2A and B). However, the restoration of BTG2 expression did not notably change tumor cell proliferation and apoptosis in vitro (data not shown). 


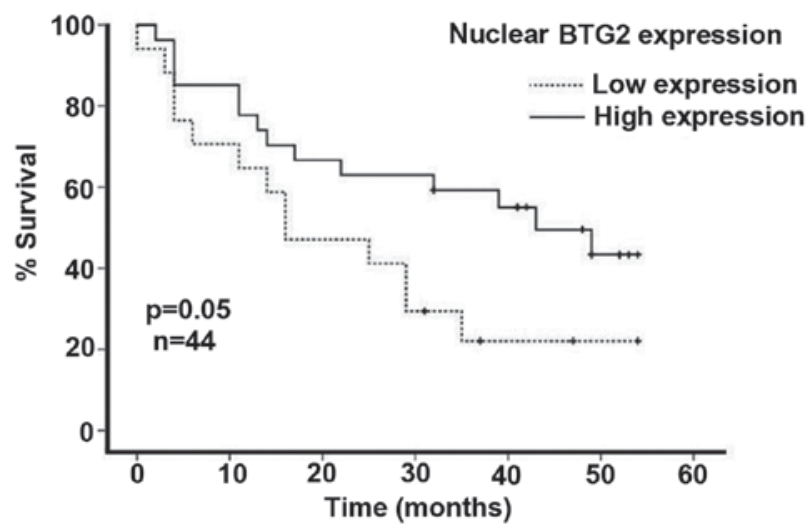

Figure 1. Kaplan-Meier curves of the overall survival of hepatocellular carcinoma (HCC) patients stratified by B-cell translocation gene 2 (BTG2) expression. HCC tissues were collected from 44 patients and immunostained for BTG2 expression. The survival rate percentage of patients with low vs. high nuclear BTG2 expression was plotted using Kaplan-Meier curves $(\mathrm{P}=0.05, \mathrm{n}=44)$.

A

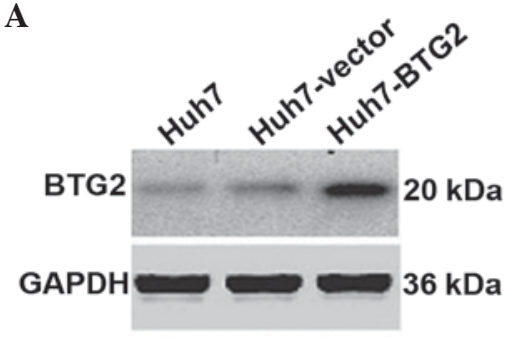

B

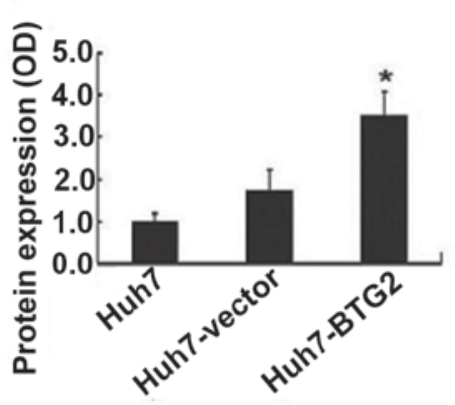

C

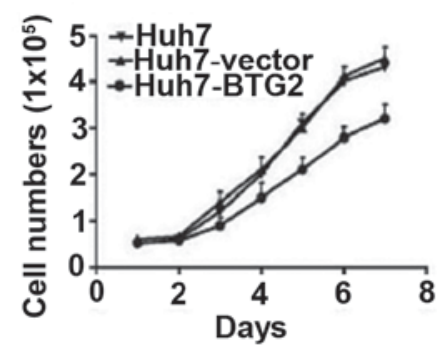

D

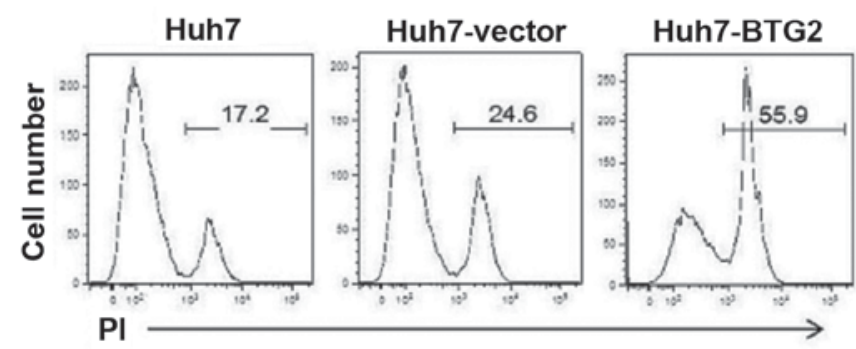

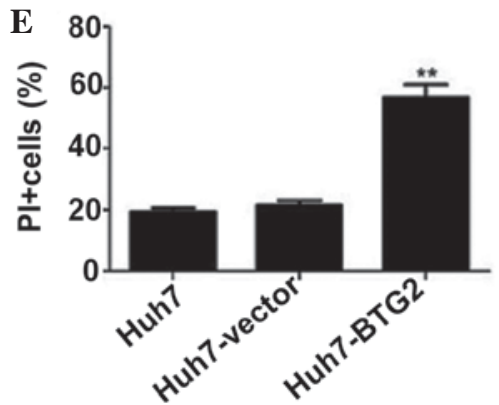

Figure 2. B-cell translocation gene 2 (BTG2) expression sensitizes radiation-induced apoptosis in Huh7 cells. (A and B, western blot analysis) Stable BTG2 overexpressed Huh7 cells were subjected to western blot analysis of BTG2 and GAPDH protein expression. The graph shows the quantitative data of the western blots analyzed by ImageJ software, and relative BTG2 protein levels were calculated from the pooled data of two independent experiments. (C, Cell proliferation assay) Cells (initial cell number $=5 \times 10^{4}$ cells per well for each cell line) were cultured and irradiated at 8 Gy, then cell numbers were counted in triplicate every day following radiation. Each data point represents three independent experiments $(\mathrm{P}<0.05)$. (D and E, Flow cytometric apoptosis assay) Cells were grown and irradiated. After $24 \mathrm{~h}$, cells were collected and subjected to apoptosis assay ( $\mathrm{P}<0.05$ and $\left.{ }^{* *} \mathrm{P}<0.01\right)$.

Next, we explored the effects of BTG2 expression on the regulation of radiation efficiency in Huh7 cells and observed that radiation treatment significantly reduced the number of Huh7-BTG2 cells compared with Huh7-vector cells. Radiation treatment did not have any effect on Huh7-vector cell proliferation compared with parental Huh7 cells (Fig. 2C).

We then assessed the effects of radiation treatment on apoptosis in Huh7-BTG2 cells compared with Huh7-vector cells. Our results revealed a marked increase $(\sim 2.5$-fold $)$ in the apoptosis of Huh7-BTG2 cells (55.9\%) following radiation treatment, compared with Huh7-vector cells (24.6\%). There was no difference in apoptosis in Huh7-vector cells (24.6\%) compared with Huh7 cells (17.2\%; Fig. 2D and E). These results demonstrate that under radiotherapy, BTG2 expression sensitized tumor cells to radiation and promoted Huh7 cell apoptosis.

BTG2 overexpression sensitizes Huh7 cells to radiation-induced apoptosis in vivo. To explore whether overexpression of BTG2 proteins represses the formation and growth of HCC cell xenografts in vivo, we transplanted hepatoma cells into nude mice as previously described (24). Our data revealed that overexpression of BTG2 per se did not have a notable effect on tumor growth in nude mice 21 days after tumor injection (Fig. 3A). We then irradiated the mice on days 21,24 and 27 (for a total of three times), and monitored the tumor volume. Data revealed that after three sessions of irradiation, the tumor volume was significantly reduced in 
A

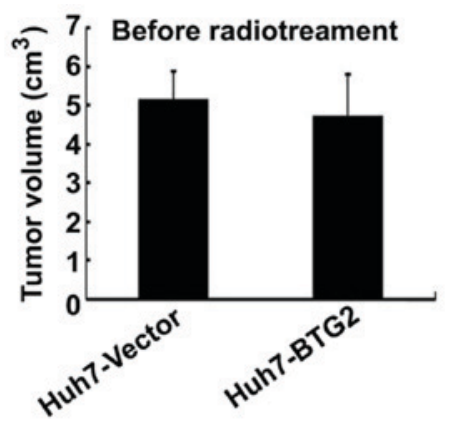

B

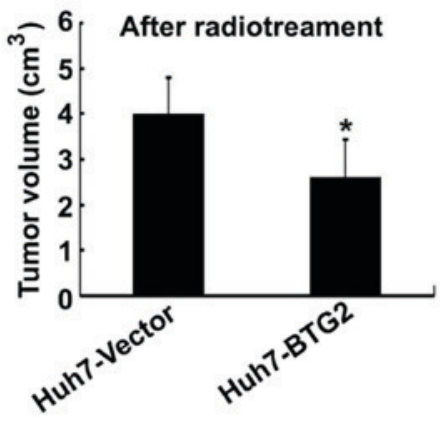

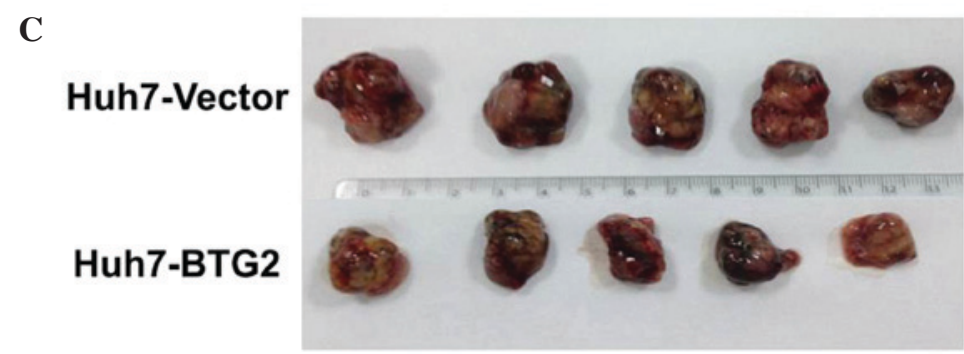

Figure 3. B-cell translocation gene 2 (BTG2) overexpression sensitizes radiation-repressed transplanted hematoma tumor growth in vivo. Cells (1x10 ${ }^{7}$ per mouse) were subcutaneously injected into the flank region of nude mice ( $n=5$ per each group). (A) Tumor volume at day 21 is shown. On days 21,24 and 27, mice were irradiated and tumor volume was monitored. (B and C) Tumor volume after day 30 is shown. Images of tumors are from a representative experiment $\left({ }^{*} \mathrm{P}<0.05, \mathrm{n}=5\right)$.

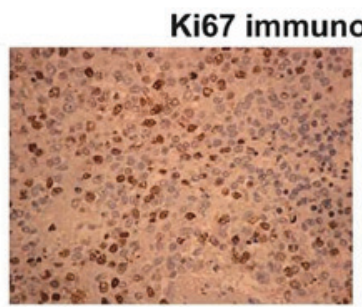

Huh7-Vector

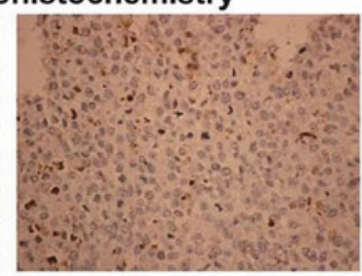

Huh7-BTG2
B

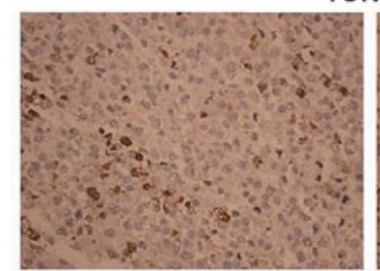

Huh7-Vector
TUNEL

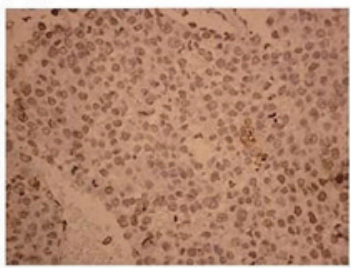

Huh7-BTG2
C

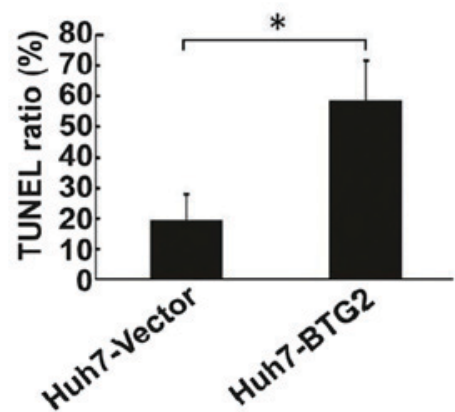

D

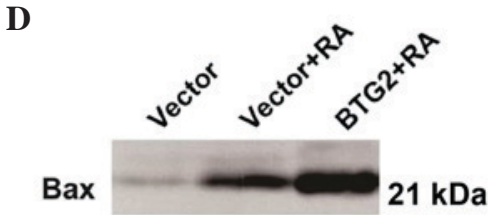

$\beta$-actin

$43 \mathrm{kDa}$

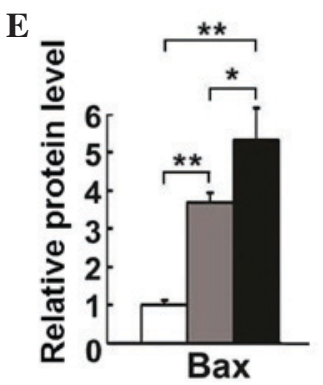

Figure 4. B-cell translocation gene 2 (BTG2) overexpression sensitize radiation-induced transplanted hematoma tumor apoptosis in vivo. Paraffin-embedded tumor xenograft sections were immunostained for Ki67 (A) and terminal deoxynucleotidyl transferase-mediated dUTP nick-end labeling (B and C). (D and E, western blot analysis) Protein lysate from tumor xenograft tissues were used for Bax immunoblotting. Relative protein levels were quantified and pooled data from two independent experiments are shown $\left({ }^{*} \mathrm{P}<0.05,{ }^{* *} \mathrm{P}<0.01\right)$.

nude mice injected with Huh7-BTG2 cells compared with those injected with Huh7-vector cells (35\% of the control, $\mathrm{P}<0.05$; Fig. 3B and C). These results suggest that BTG2 overexpression reduced hepatoma tumor growth following radiation therapy in nude mice.

Furthermore, we resected xenograft tissues and stained the xenograft sections for H\&E, Ki67, BTG2 and TUNEL. Our data revealed that following radiation treatment, the growth of tumor xenografts was significantly repressed in BTG2-overexpressed hepatoma tumors compared with vector-only hepatoma cell injection, as evident from immunohistochemistry staining with Ki67 (a proliferation marker) (Fig. 4A). A marked increase in TUNEL staining was observed in BTG2-overexpressing tumor sections (39.8\%, $\mathrm{P}<0.05$; Fig. 4B and $\mathrm{C}$ ), as compared 
with vector-expressing tumor sections. Bax expression was also significantly increased in tumor tissues with BTG2 overexpression, compared with the group that had no or low BTG2 expression (1.5-fold, $\mathrm{P}<0.05$; Fig. 4D and $\mathrm{E}$ ). These results provide evidence that BTG2 overexpression sensitized HCC cells in vivo to irradiation treatment-induced apoptosis.

\section{Discussion}

In the present study, we first associated BTG2 expression with HCC patient survival, and restored BTG2 expression in HCC cells to assess its effects in HCC cells in vitro and in vivo. We demonstrated that BTG2 expression was associated with the prognosis of hepatoma patients, and that overexpression of BTG2 proteins sensitized radiation-induced apoptosis in vitro and in vivo, although overexpression of BTG2 protein per se did not affect HCC cell phenotypes. Future studies would confirm BTG2 as a prognostic marker for HCC patients, and further investigate the usefulness of BTG2 as a therapeutic target for HCC patients.

BTG2 is a member of the anti-proliferative family of proteins (25), and plays an essential role in regulating cell proliferation, apoptosis and DNA repair $(13,26,27)$. Several previous studies have reported that BTG2 expression was reduced in various human cancers including renal cell carcinoma, prostate cancer, gastric cancer, melanoma, breast cancer and lung cancer $(8,16-20)$. Further studies also revealed that BTG2 expression was reversely associated with tumor grade, size, metastasis, recurrence and survival in breast cancer $(8,28)$. Consistent with these studies, the results from the present study also revealed markedly downregulated BTG2 expression in hepatoma tissues. Therefore, BTG 2 protein may also be a potential tumor suppressor in human liver cancer.

Furthermore, we verified the function of BTG2 proteins in human liver cancer cells by restoring BTG2 expression. Our in vitro and in vivo results indicate that BTG2 overexpression could sensitize irradiation-induced HCC cell apoptosis. These data suggest a strong crosstalk between BTG2 signaling and radiation in apoptosis induction. Further studies are required to unravel this crosstalk and underlying molecular mechanisms, and the results may be helpful in designing combination therapies for hepatic tumors.

Indeed, the dose and duration of radiotherapy are significant factors in achieving optimal therapeutic success. In the present study, we only used and combined one dose and a specific time point to irradiate HCC cells in vitro and in vivo. Thus, future investigation of optimal doses and duration for radiotherapy in BTG2-overexpressed HCC cells would provide insightful data before its potential use in a patient care setting. The present study provided evidence that BTG2 expression was associated with better HCC patient survival, and that overexpression of BTG2 in hepatic tumor cells sensitized tumor cell susceptibility to irradiation in vitro and in vivo. However, this study is just a proof-of-principle, and further study should be carried out before these findings can be translated into clinical practice.

\section{Acknowledgements}

The authors would like to thank Dr. Ravinder Kumar Kaundal of the Department of Immunobiology, Yale School of Medicine for his help in the preparation of this manuscript. This study was supported in part by grants from the National Natural Science Foundation of China (nos. 81272498, 30973457, 30901764 and 31400770).

\section{References}

1. Jemal A, Bray F, Center MM, Ferlay J, Ward E and Forman D: Global cancer statistics. CA Cancer J Clin 61: 69-90, 2011.

2. Hu YJ, Li HY, Qiu KJ, Li DC, Zhou JH, Hu YH and Zhang FM: Downregulation of Notch1 inhibits the invasion of human hepatocellular carcinoma HepG2 and MHCC97H cells through the regulation of PTEN and FAK. Int J Mol Med 34: 1081-1086, 2014.

3. Liu W, Xu C, Wan H, Liu C, Wen $\mathrm{C}$, Lu H and Wan F: MicroRNA-206 overexpression promotes apoptosis, induces cell cycle arrest and inhibits the migration of human hepatocellular carcinoma HepG2 cells. Int J Mol Med 34: 420-428, 2014.

4. Krishnan S, Dawson LA, Seong J, Akine Y, Beddar S, Briere TM, Crane $\mathrm{CH}$ and Mornex F: Radiotherapy for hepatocellular carcinoma: an overview. Ann Surg Oncol 15: 1015-1024, 2008.

5. Hawkins MA and Dawson LA: Radiation therapy for hepatocellular carcinoma: from palliation to cure. Cancer 106: 1653-1663, 2006.

6. Liu S, Chen P, Hu M, Tao Y, Chen L, Liu H, Wang J, Luo J and Gao G: Randomized, controlled phase II study of post-surgery radiotherapy combined with recombinant adenoviral human p53 gene therapy in treatment of oral cancer. Cancer Gene Ther 20: 375-378, 2013.

7. Zhao J, Liu C and Zhao Z: ARID1A: a potential prognostic factor for breast cancer. Tumour Biol 35: 4813-4819, 2014.

8. Rouault JP, Falette N, Guéhenneux F, Guillot C, Rimokh R, Wang Q, Berthet C, Moyret-Lalle C, Savatier P, Pain B, et al: Identification of BTG2, an antiproliferative p53-dependent component of the DNA damage cellular response pathway. Nat Genet 14: 482-486, 1996.

9. Mao B, Zhang Z and Wang G: BTG2: a rising star of tumor suppressors (review). Int J Oncol 46: 459-464, 2015.

10. He Z, Liu Y, Xiao B and Qian X: miR-25 modulates NSCLC cell radio-sensitivity through directly inhibiting BTG2 expression. Biochem Biophys Res Commun 457: 235-241, 2015.

11. Guehenneux F, Duret L, Callanan MB, Bouhas R, Hayette S, Berthet C, Samarut C, Rimokh R, Birot AM, Wang Q, et al: Cloning of the mouse BTG3 gene and definition of a new gene family (the BTG family) involved in the negative control of the cell cycle. Leukemia 11: 370-375, 1997.

12. Sukhatme VP, Kartha S, Toback FG, Taub R, Hoover RG and Tsai-Morris $\mathrm{CH}$ : A novel early growth response gene rapidly induced by fibroblast, epithelial cell and lymphocyte mitogens. Oncogene Res 1: 343-355, 1987.

13. Tirone F: The gene PC3(TIS21/BTG2), prototype member of the PC3/BTG/TOB family: regulator in control of cell growth, differentiation and DNA repair? J Cell Physiol 187: 155-165, 2001.

14. Duriez C, Moyret-Lalle C, Falette N, El-Ghissassi F and Puisieux A: BTG2, its family and its tutor. Bull Cancer 91: E242-E253, 2004.

15. Mauxion F, Chen CY, Séraphin B and Shyu AB: BTG/TOB factors impact deadenylases. Trends Biochem Sci 34: 640-647, 2009.

16. Ezzeddine N, Chang TC, Zhu W, Yamashita A, Chen CY, Zhong Z, Yamashita Y, Zheng D and Shyu AB: Human TOB, an antiproliferative transcription factor, is a poly(A)-binding protein-dependent positive regulator of cytoplasmic mRNA deadenylation. Mol Cell Biol 27: 7791-7801, 2007.

17. Lim IK: TIS21 (/BTG2/PC3) as a link between ageing and cancer: sell cycle regulator and endogenous cell death molecule. J Cancer Res Clin Oncol 132: 417-426, 2006.

18. Okochi K, Suzuki T, Inoue J, Matsuda S and Yamamoto T: Interaction of anti-proliferative protein Tob with poly(A)-binding protein and inducible poly(A)-binding protein: implication of Tob in translational control. Genes Cells 10: 151-163, 2005.

19. Ruan L, Osawa M, Hosoda N, Imai S, Machiyama A, Katada T, Hoshino S and Shimada I: Quantitative characterization of Tob interactions provides the thermodynamic basis for translation termination-coupled deadenylase regulation. J Biol Chem 285: 27624-27631, 2010.

20. Winkler GS: The mammalian anti-proliferative BTG/Tob protein family. J Cell Physiol 222: 66-72, 2010. 
21. Horvilleur E, Bauer M, Goldschneider D, Mergui X, de la Motte A, Bénard J, Douc-Rasy S and Cappellen D: p73alpha isoforms drive opposite transcriptional and post-transcriptional regulation of MYCN expression in neuroblastoma cells. Nucleic Acids Res 36: 4222-4232, 2008

22. Gong L, Wei LX, Ren P, Zhang WD, Liu XY, Han XJ, Yao L, Zhu SJ, Lan M, Li YH and Zhang W: Dysplastic nodules with glypican-3 positive immunostaining: a risk for early hepatocellular carcinoma. PloS One 9: e87120, 2014.

23. Qiu L, Yin G, Cheng L, Fan Y, Xiao W, Yu G, Xing M, Jia R, Sun R, Ma X, et al: Astragaloside IV ameliorates acute pancreatitis in rats by inhibiting the activation of nuclear factor- $\kappa \mathrm{B}$. Int J Mol Med 35: 625-636, 2015.

24. Magnus N, Meehan B, Garnier D, Hashemi M, Montermini L, Lee TH, Milsom C, Pawlinski R, Ohlfest J, Anderson M, et al: The contribution of tumor and host tissue factor expression to oncogene-driven gliomagenesis. Biochem Biophys Res Commun 454: 262-268, 2014.
25. Boiko AD, Porteous S, Razorenova OV, Krivokrysenko VI, Williams BR and Gudkov AV: A systematic search for downstream mediators of tumor suppressor function of p53 reveals a major role of BTG2 in suppression of Ras-induced transformation. Genes Dev 20: 236-252, 2006.

26. Chung LC, Tsui KH, Feng TH, Lee SL, Chang PL and Juang HH: L-Mimosine blocks cell proliferation via upregulation of B-cell translocation gene 2 and $\mathrm{N}$-myc downstream regulated gene 1 in prostate carcinoma cells. Am J Physiol Cell Physiol 302: C676-C685, 2012.

27. Liu M, Wu H, Liu T, Li Y, Wang F, Wan H, Li X and Tang H: Regulation of the cell cycle gene, BTG2, by miR-21 in human laryngeal carcinoma. Cell Res 19: 828-837, 2009.

28. Zhao Z, Lu P, Zhang H, Xu H, Gao N, Li M and Liu C: Nestin positively regulates the $\mathrm{Wnt} / \beta$-catenin pathway and the proliferation, survival and invasiveness of breast cancer stem cells. Breast Cancer Res 16: 408, 2014. 